\title{
Modélisation de l'absorption et de la désorption de l'hydrogène dans un réservoir à hydrure de magnésium activé
}

\author{
Albin Chaise $^{1, a}$, Philippe Marty ${ }^{2}$, Patricia De Rango ${ }^{1}$ et Daniel Fruchart ${ }^{1}$ \\ 1 Institut NEEL-CRETA, 25 avenue des Martyrs, BP 166, 38042 Grenoble Cedex 9, France \\ 2 LEGI, Domaine Universitaire, BP 53, 38041 Grenoble Cedex 9, France
}

Reçu le 15 mars 2007, accepté le 7 juin 2007

\begin{abstract}
Résumé - Afin de démontrer la faisabilité du stockage de l'hydrogène grâce à l'hydrure de magnésium activé, un réservoir de $250 \mathrm{~cm}^{3}$ contenant $120 \mathrm{~g}$ d'hydrure a été réalisé. Le magnésium réagit avec l'hydrogène de façon réversible et absorbe jusqu'à 7,6 \% massique. Le fonctionnement de ce réservoir fait l'objet de cette étude. Le logiciel Fluent a été utilisé pour le modéliser numériquement. Le code s'appuie sur des conditions aux limites et certaines données thermiques déduites de l'expérience. La pression est considérée comme homogène dans le réservoir. Les simulations sont en accord avec l'expérience dans les domaines de température et de pression favorables à une charge ou une décharge du réservoir rapide.
\end{abstract}

Mots clés : Stockage / hydrogène / hydrure de magnésium / simulation / réservoir

\begin{abstract}
Numerical study of heat and mass transfer in a doped-magnesium hybride tank. In order to appraise the faisability of hydrogen storage in activated magnesium hydride, a $250 \mathrm{~cm}^{3}$ tank containing $120 \mathrm{~g}$ of hydrid has been built. Up to $7.6 \mathrm{wt} \%$ hydrogen can be stored reversibly in magnesium hydrid. The charging and discharging process is studied here. Fluent software has been used for tank numerical modeling. Boundary conditions and some thermal data are taken from experimental results. Pressure is considered to be homogeneous in the tank. Simulations fit experimental results when temperature and pressure conditions allow charge and discharge to be fast.
\end{abstract}

Key words: Hydrogen storage / magnesium hybride / numerical modeling

\section{Introduction}

L'hydrogène est un vecteur d'énergie prometteur. Sa combustion très énergétique ne libère que de l'eau. Néanmoins, le développement de son utilisation par couplage avec des piles à combustible ou des moteurs thermiques nécessite de pouvoir stocker ce gaz de façon efficace. Trois modes de stockage sont envisagés actuellement : hyperbare (700 bar), liquide à basse température $(20 \mathrm{~K})$, et solide sous forme d'hydrure métallique ou d'hydrure complexes réversibles. Le stockage sous forme d'hydrure métallique apparaît avantageux du point de vue de la sûreté (décharge endothermique, pression modérée) avec une densité volumique d'hydrogène théoriquement supérieure à celle de l'hydrogène liquide.

\footnotetext{
a Auteur correspondant :

albin.chaise@grenoble.cnrs.fr
}

Cette étude se base sur le magnésium en tant que matériau de stockage réversible. L'hydrure de magnésium contient jusqu'à 7,6 \% massique d'hydrogène. Les cinétiques de réaction du magnésium avec l'hydrogène sont rapides grâce à une élaboration de la poudre d'hydrure par cobroyage avec des éléments de transition. La réaction d'absorption (resp. de désorption) de l'hydrogène est fortement exothermique (resp. endothermique). Ces réactions ont lieu à des pressions inférieures à $1 \mathrm{MPa}$ et à des températures comprises entre 200 et $350{ }^{\circ} \mathrm{C}$.

Afin de démontrer la possibilité d'utiliser l'hydrure de magnésium activé pour le stockage de l'hydrogène à grande échelle, un premier réservoir à hydrure de magnésium a été conçu et réalisé par l'Institut Néel. Son étude montre qu'il existe un couplage très fort entre le dégagement ou la consommation de chaleur et les cinétiques de réactions. Le réservoir possède donc un fonctionnement complexe qu'il nous a paru intéressant de 


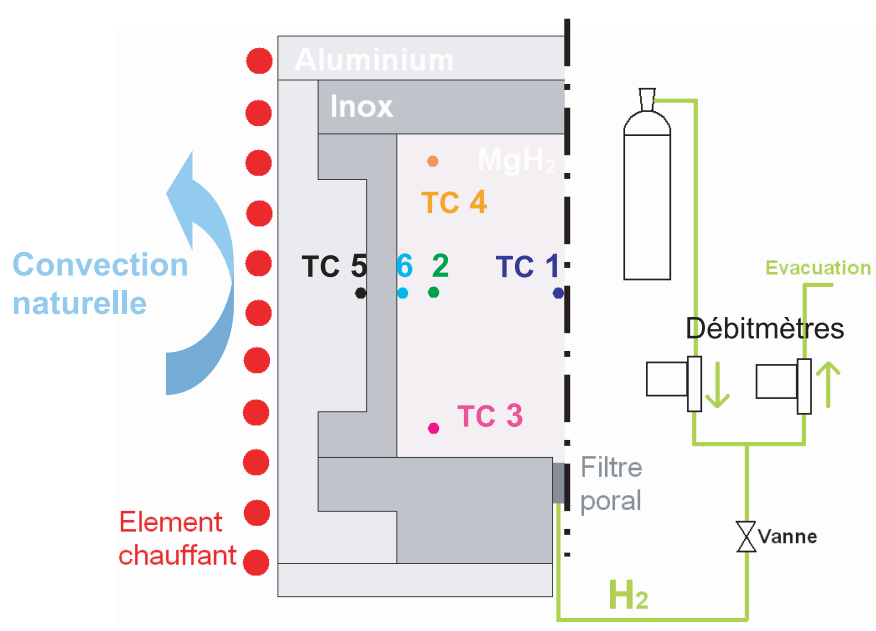

Fig. 1. Schéma du réservoir et de son alimentation en hydrogène.

comprendre en réalisant un modèle numérique. Le logiciel commercial Fluent a été utilisé pour cette étude.

\section{Description de l'expérience et du code}

La plupart des réservoirs à hydrure étudiés sont destinés à recevoir l'alliage $\mathrm{LaNi}_{5}[1,2]$. Généralement les recherches menées sur ces matériaux sont découplés de celles menées sur la modélisation de réservoir $[3,4]$. Dans le cas de l'hydrure de magnésium, une connaissance approfondie du matériau apparaît primordiale dans l'optique de la conception d'un réservoir. Les conditions d'élaboration du $\mathrm{MgH}_{2}$ activé par ball-milling et les propriétés d'absorption de ces poudres ont fait l'objet d'une étude préalable [5]. Ces poudres sont maintenant produites à l'échelle semi-industrielle (MCP Mg-Serbien à Roman sur Isère) avec de très bonnes performances en termes de cinétique d'absorption-désorption, et ont permis le développement d'un premier prototype de réservoir à base d'hydrure de magnésium.

\subsection{Configuration du réservoir}

Le réservoir de $250 \mathrm{~cm}^{3}$ contient $120 \mathrm{~g}$ d'hydrure sous forme de poudre (70 $\mathrm{Nl}$ d'hydrogène). Il est équipé de 6 thermocouples, d'un capteur de pression et de deux débitmètres (entrée et sortie de gaz) (voir Fig. 1). Le réservoir est entouré d'une coque d'aluminium qui uniformise sa température et d'un four qui maintient cette température constante. Il est refroidi par la convection naturelle de l'air autour de l'ensemble.

La charge « classique »du réservoir consiste à porter le réservoir déchargé à $300{ }^{\circ} \mathrm{C}$ avec une pression inférieure à la pression d'équilibre. Lorsque la température est homogène, une pression d'alimentation de $0,8 \mathrm{MPa}$ est appliquée à $t=0$. La réaction d'hydruration étant très exothermique $\left(\Delta H=76700 \mathrm{~J} \cdot \mathrm{mol}_{\mathrm{H} 2}^{-1}\right)$ la température s'élève rapidement et atteint la température d'équilibre

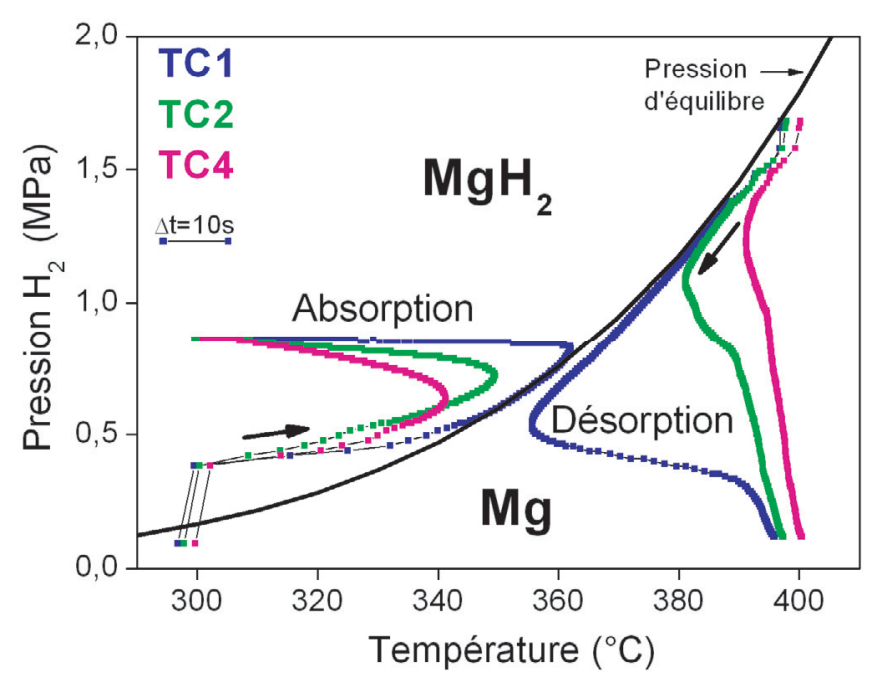

Fig. 2. Évolution de la pression et de la température au cours d'une charge et d'une décharge.

ce qui bloque la réaction (Fig. 2). Le refroidissement du réservoir s'avère nécessaire pour permettre au chargement de se poursuivre. La vitesse et la durée de chargement apparaissent directement corrélées à l'efficacité du refroidissement. La pression à l'intérieur du réservoir évolue lentement en fonction du débit entrant et atteint la pression d'alimentation en fin de réaction. En effet, le système de conduite d'hydrogène comporte des pertes de charges importantes dues notamment au poral qui empêche la poudre fine $(5 \mu \mathrm{m})$ de sortir du réservoir.

La décharge classique du réservoir consiste à porter le réservoir chargé à $400^{\circ} \mathrm{C}$ avec une pression supérieure à la pression d'équilibre $(0,6 \mathrm{MPa})$. À $t=0$ les vannes sont ouvertes et l'hydrogène s'échappe à la pression ambiante. La réaction de désorption étant endothermique, la température s'abaisse et atteint l'équilibre. Il faut alors fournir de la chaleur au réservoir pour permettre à la réaction de se poursuivre.

\subsection{Détermination des conditions limites et des paramètres thermiques}

Si les caractéristiques thermodynamiques et cinétiques de la réaction d'hydruration ont été étudiées [6], les paramètres thermiques du matériau sont à déterminer. Ils sont fonctions de la compacité, de la température, de la pression d'hydrogène et du degré d'hydruration de la poudre. Une bonne connaissance de la conductivité thermique est particulièrement nécessaire à la pertinence d'une simulation. Cette valeur a été obtenue pour l'hydrure à $0,5 \mathrm{MPa}$ par ajustement de ce paramètre dans la simulation purement thermique d'une expérience de montée en température (voir Fig. 3). Cette valeur est en accord avec la littérature [7]. De cette expérience, une valeur de résistance de paroi est également extraite.

Les pertes thermiques du réservoir conditionnent à l'absorption le temps de chargement. Leurs déterminations en fonction de la température interne du 

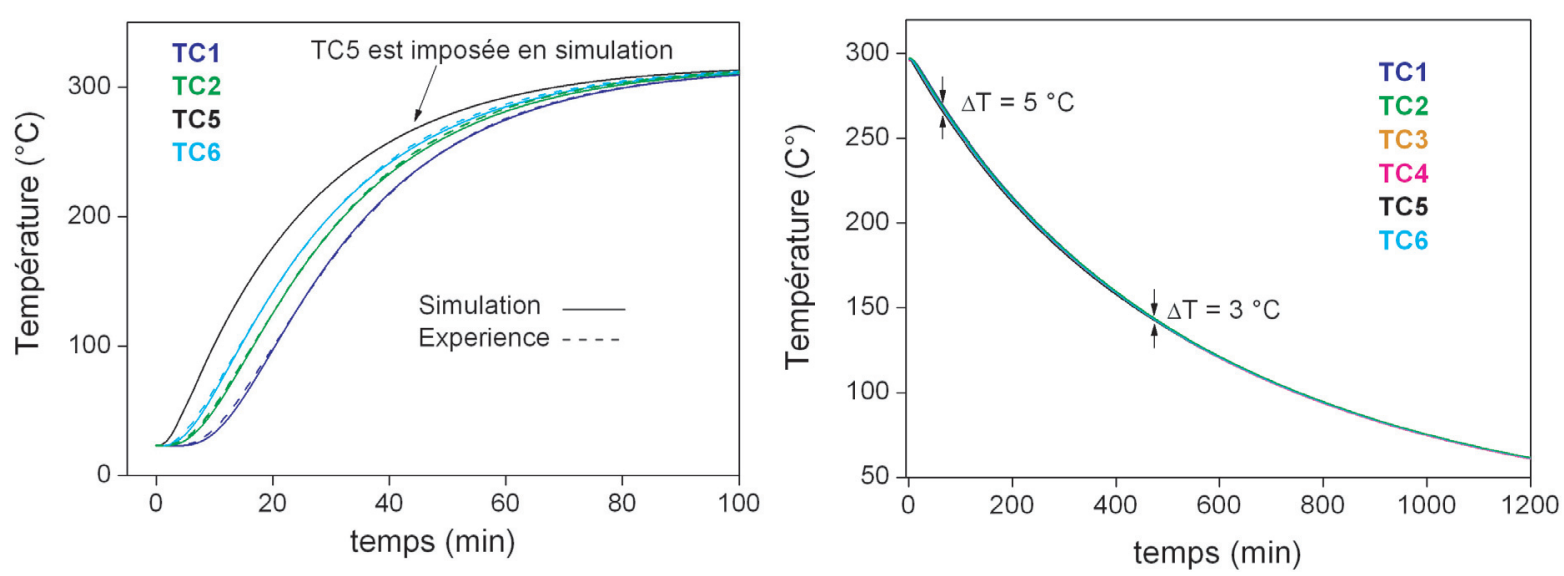

Fig. 3. Montée en température du réservoir simulée et expérimentale. Descente en température du réservoir expérimentale.

réservoir est nécessaire. Lors d'un refroidissement libre du réservoir, les températures en tous points de celui-ci ne diffèrent que de quelques degrés (Fig. 3). De la vitesse de refroidissement, sont extraites les pertes thermiques en fonction de la température interne.

\subsection{Le code de calcul et la pression}

Le logiciel commercial Fluent a été utilisé pour développer un modèle numérique de ce réservoir. Le modèle est $2 \mathrm{D}$ axisymétrique. Les équations résolues sont celles de la chaleur où le terme source représente l'exoendothermicité de la réaction. Ce terme source dépend de la vitesse d'hydruration. Cette vitesse d'hydruration est calculée par une fonction de Fluent (UDF : User Define Function) où sont déterminés tous les paramètres réactionnels (pression d'équilibre, cinétique, degré d'hydruration...).

La poudre d'hydrure, dont la porosité est grande $(\varepsilon=0,7)$ n'offre qu'une résistance négligeable au passage de l'hydrogène. À tout instant, la pression peut donc être considérée comme homogène dans le réservoir. La circulation d'hydrogène dans le matériau poreux et ses effets sont négligés. La poudre et le gaz sont traités comme un seul matériau massif. La pression est une nouvelle variable gérée par l'UDF : à chaque pas de temps, la pression est déduite d'un bilan de masse d'hydrogène entrante, consommée et présente dans le réservoir. L'influence négligeable du transfert de masse dans le réservoir est vérifié avec le développement d'un modèle tenant compte de la circulation d'un gaz dans un milieu poreux.

Les paramètres globaux $\lambda, C_{\mathrm{p}}$ et $\rho$ sont ceux de l'ensemble poudre et gaz. Seul $\lambda$ dépend du degré d'hydruration de la poudre.

$$
\begin{aligned}
\rho C_{\mathrm{p}} \frac{\partial T}{\partial t} & =\operatorname{div}(\lambda \operatorname{grad}(T))+s \\
s & =\rho \tau \frac{\partial x}{\partial t} \Delta H \\
\frac{\mathrm{d} x}{\mathrm{~d} t} & =f\left(P_{\mathrm{eq}}, T\right) \quad P_{\mathrm{eq}}=f(T) \quad \lambda=f(x)
\end{aligned}
$$

$\rho:$ masse volumique

$C_{\mathrm{p}}$ : capacité calorifique massique

$\lambda$ : conductivité thermique

$\tau$ : taux massique maximal d'hydrogène de l'hydrure

$x$ : degré d'hydruration du matériau

$\Delta H$ : enthalpie massique de la réaction

$P_{\text {eq }}:$ pression d'équilibre de la réaction

$t$ : temps

$T$ : température

\section{Résultats : comparaison expérimental-numérique}

Le calcul est stable pour des pas de temps inférieurs à $1 \mathrm{~s}$. Les résultats sont indépendants du pas de temps au dessous de $0,01 \mathrm{~s}$ et indépendants du maillage pour 50 mailles. $\mathrm{mm}^{-2}$. Il est toutefois nécessaire d'effectuer les calculs en double précision.

\subsection{L'absorption}

La charge du réservoir a été simulée pour des conditions identiques aux conditions expérimentales. La figure $4 \mathrm{a}$ correspondant au chargement du réservoir, présente l'évolution de la température au centre (TC1) et en périphérie du réservoir (TC4), ainsi que l'évolution de la pression et du volume absorbé.

L'expérience et la simulation présentent un bon accord. Le temps de chargement est notamment assez précis, preuve que la thermique du système est représentative de la réalité. Le volume absorbé expérimentalement présente une rupture de pente après $60 \mathrm{~min}$. Ceci provient du débitmètre qui présente un seuil de coupure pour de faibles débits.

Les écarts observés entre les valeurs expérimentales et calculées ont été attribués au fait que la température et la pression sont proches des conditions d'équilibre durant la plus grande partie de la charge (voir Fig. 2). Or les cinétiques sont mal connues dans de telles conditions car difficilement mesurables. Les lois cinétiques sont donc 
(a)

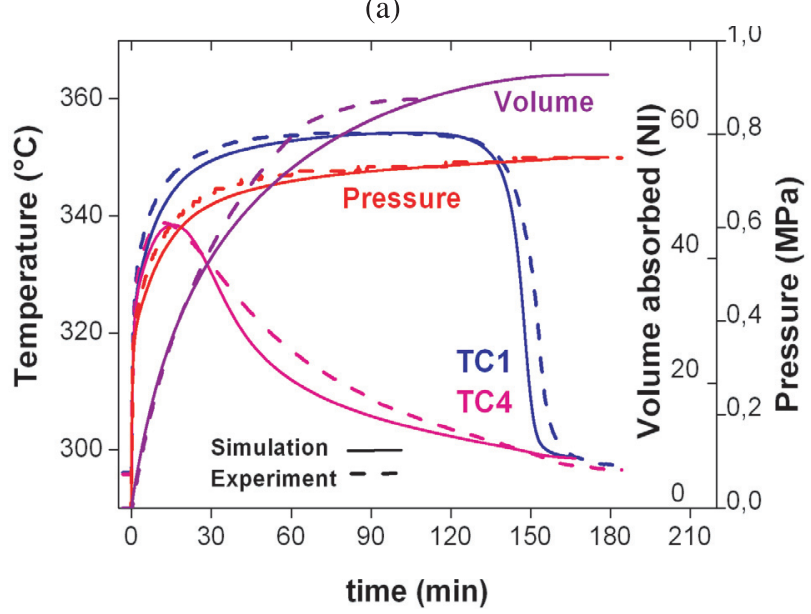

(b)

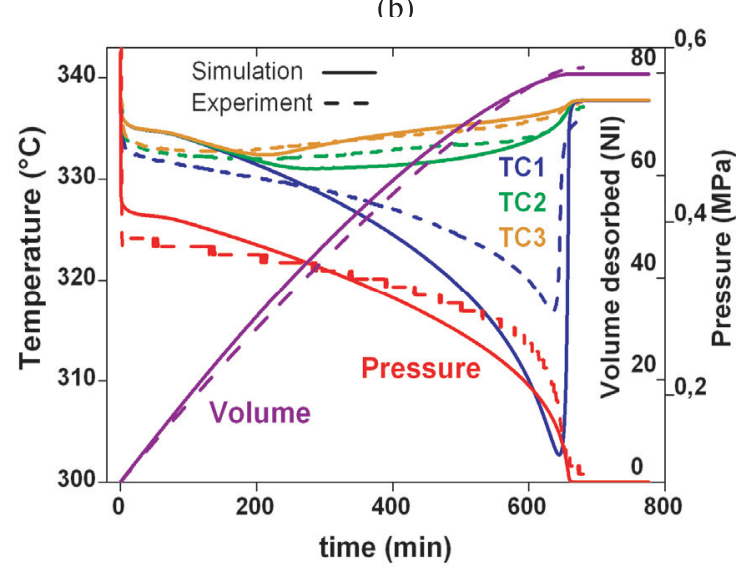

Fig. 4. (a) Charge du réservoir à $300{ }^{\circ} \mathrm{C} 0,8 \mathrm{MPa}$ simulée et expérimentale. (b) Décharge à $340{ }^{\circ} \mathrm{C} 0,1 \mathrm{MPa}$ simulée et expérimentale.

extrapolées dans ce domaine de faible réactivité, et les températures ne sont pas toujours fidèles à la réalité.

\subsection{La désorption}

La décharge du réservoir a été simulée (Fig. 4b) pour des conditions identiques aux conditions expérimentales. La vitesse de déchargement est limitée par les pertes de charge importantes dans le poral qui maintient la pression dans le réservoir à une valeur élevée et proche de l'équilibre. Les temps de déchargement sont donc bien plus longs que les temps de chargement.

La simulation de la désorption est délicate car les cinétiques de cette réaction sont mal connues pour des pressions autres que des pressions faibles (20 mbar). L'évolution des températures et de la pression n'est donc pas totalement concordante avec l'expérience. En revanche, la durée de déchargement est réaliste.

\section{Conclusion}

Grâce à l'étude conjointe du matériau et du réservoir, une modélisation cohérente a pu être réalisée. Les études cinétiques et thermodynamiques du matériau ainsi que les conditions limites extraites d'un réservoir réel ont permis de faire converger l'expérience et la simulation. L'importance de la thermique du réservoir a été mise en lumière. Une plus grande conductivité thermique de la poudre, de faibles résistances de paroi et une grande capacité d'échange thermique sont nécessaires à l'obtention de performances satisfaisantes notamment en termes de réduction du temps de chargement. La réduction importante des pertes de charge est nécessaire afin d'assurer une plus faible différence de pression entre l'alimentation et l'intérieur du réservoir.

Enfin, l'étude des lois cinétiques doit être approfondie, en particulier dans des conditions proches de l'équilibre réactionnel, puisque nous avons constaté que les zones du réservoir les plus éloignées des parties susceptibles de fournir ou d'évacuer la chaleur évoluaient très vite vers les conditions d'équilibre.

Ce modèle numérique sera par la suite utilisé pour concevoir un réservoir de plus grande capacité dont les performances en termes de poids, de vitesse de chargement et de rendement thermique seront les meilleures possibles.

\section{Références}

[1] A. Demircan, M. Demiralp, Y. Kaplan, M.D. Mat, T.N. Veziroglu, Experimental and theoretical analysis of hydrogen absorption in $\mathrm{LaNi}_{5}-\mathrm{H}_{2}$ reactors, Int. J. Hydrogen Energy 30 (2005) 1437-1446

[2] M. Botzung, S. Chaudourne, C Perret, M. Latroche, A. Percheron-Guegan, P. Marty, WHEC 16/13-16 June 2006, Lyon France, Hydrogen storage using metal hydrides in a stationary cogeneration system, 2006

[3] F. Askri, A. Jemni, S. Ben Nasrallah, Study of two dimensional and dynamic heat and mass transfer in metalhydrogen reactor, Int. J. Hydrogen Energy 28 (2003) 537-557

[4] A. Dogan, Y. Kaplan, T.N. Veziroglu, Numerical investigaton of heat and mass transfer in a metal hydride bed, Appl. Math. Computation 150 (2004) 169-180

[5] P. de Rango, A. Chaise, J. Charbonnier, D. Fruchart, M. Jehan, P. Marty, S. Miraglia, S. Rivoirard, N. Skryabina, 2007 Nanostructured magnesium hydride for pilot tank development, J. Alloys Comp. 2007, in press

[6] G. Linag, J. Huot, S. Boily, Avan Neste, R. Schulz, J. Alloys Comp. 291 (1999) 295

[7] E. Hahne, J. Kallweit Thermal conductivity of metal hydride material for storage of hydrogen: experimental investigation, Int. J. Hydogen Energy 23 (1998) 107-114 